\title{
A numerical model of the ionospheric signatures of time-varying magnetic reconnection: III. Quasi-instantaneous convection responses in the Cowley-Lockwood paradigm
}

\author{
S. K. Morley ${ }^{1, *}$ and M. Lockwood ${ }^{1,2}$ \\ ${ }^{1}$ School of Physics and Astronomy, University of Southampton, UK \\ *also at: CRC for Satellite Systems, University of Newcastle, NSW, Australia \\ ${ }^{2}$ Rutherford Appleton Laboratory, Chilton, Oxfordshire, UK
}

Received: 7 October 2005 - Revised: 18 January 2006 - Accepted: 13 March 2006 - Published: 19 May 2006

\begin{abstract}
Using a numerical implementation of the Cowley and Lockwood (1992) model of flow excitation in the magnetosphere-ionosphere (MI) system, we show that both an expanding (on a $\sim 12$-min timescale) and a quasiinstantaneous response in ionospheric convection to the onset of magnetopause reconnection can be accommodated by the Cowley-Lockwood conceptual framework. This model has a key feature of time dependence, necessarily considering the history of the coupled MI system. We show that a residual flow, driven by prior magnetopause reconnection, can produce a quasi-instantaneous global ionospheric convection response; perturbations from an equilibrium state may also be present from tail reconnection, which will superpose constructively to give a similar effect. On the other hand, when the MI system is relatively free of pre-existing flow, we can most clearly see the expanding nature of the response. As the open-closed field line boundary will frequently be in motion from such prior reconnection (both at the dayside magnetopause and in the cross-tail current sheet), it is expected that there will usually be some level of combined response to dayside reconnection.
\end{abstract}

Keywords. Ionosphere (Modeling and forecasting; Plasma convection) - Magnetospheric physics (Magnetosphereionosphere interactions)

\section{Introduction}

1.1 Observations of expanding and quasi-instantaneous convection responses

There are seemingly conflicting views on how the ionospheric convection pattern responds to a change in the applied reconnection. The first observation of an evolving

Correspondence to: S. K. Morley

(steven.morley@newcastle.edu.au) ionospheric response to magnetopause reconnection was made by Lockwood et al. (1986) who correlated IMF data from the AMPTE-UKS (Active Magnetospheric Particle Tracer Explorer - U.K. Satellite) with ion temperature measurements from EISCAT. They found that the observed ion temperature enhancement propagated away from noon with a mean velocity of $2.6 \pm 0.3 \mathrm{~km} \mathrm{~s}^{-1}$. Other studies, for example, the case study by Saunders et al. (1992) and the statistical surveys by Todd et al. (1988), Etemadi et al. (1988) and Khan and Cowley (1999) all reported this expansion, which was combined with ideas on boundary motions and flows discussed previously by Siscoe and Huang (1985) and Freeman and Southwood (1988) in the model of the ionospheric flow response proposed by Cowley and Lockwood (1992). The Cowley-Lockwood model invokes an equilibrium state that the near-Earth system will approach with the cessation of reconnection. As the system approaches equilibrium the flows will die away, even if open flux is still present. Subsequent reconnection at the magnetopause or in the tail will perturb the system away from equilibrium and excite flow which carries the system towards a new equilibrium for the changed amount of open flux. Under an applied change in magnetopause reconnection, convection will first respond locally on the dayside, before evolving around the flanks and into the nightside (Cowley and Lockwood, 1997).

The view of an expanding reconfiguration of the convection flow pattern, interpreted in the framework of the Cowley-Lockwood model, was challenged by Ridley et al. (1998). Their study used a global assimilative mapping technique (AMIE, Richmond and Kamide, 1988) to generate patterns of electrostatic potential from magnetometer data. A method of producing residual potential patterns, subtracting an initial potential map from subsequent potential maps, was developed to show the deviation from the initial potential pattern. They interpreted their results as showing a globally instantaneous response, within the temporal resolution of their data, and thus concluded that this was inconsistent with the

Published by Copernicus GmbH on behalf of the European Geosciences Union. 
Cowley-Lockwood model. Ruohoniemi and Baker (1998) used another large-scale technique to observe the convection response to variations in the IMF. Using the Doppler shift of observed coherent radar echoes from the SuperDARN radar network, these authors then used the line-of-sight velocities to constrain a mathematical fitting of an electrostatic potential pattern. Where insufficient data is gathered for a spherical harmonic fit of a given order, data from a statistical model (the APL model, see Ruohoniemi and Baker (1998) and references therein) is used to augment the measurements. The same technique was used by Ruohoniemi and Greenwald (1998) for a more detailed examination of one of the periods used as a case study in Ruohoniemi and Baker (1998). These studies also concluded that the ionospheric convection pattern responded globally simultaneously to a change in the applied reconnection voltage.

In a comment on the Ridley et al. (1998) paper, Lockwood and Cowley (1999) argued that the data presented by Ridley et al. (1998) in fact showed an expansion commensurate with the timescales for reconfiguration inferred by the prior, directly-measured studies. They also raised an important point regarding the distinction between the convection change and the shape of the convection pattern. A recent review by Ruohoniemi et al. (2002) highlighted some further shortcomings of using global mapping procedures (e.g. AMIE). These authors reached the conclusion that global assimilative techniques have a tendency to "globalize" local behaviour. They noted that the integration of statistical model data into the solution can also tend to globalize the effects of a local disturbance. They considered the case of a localized velocity vortex and reasoned that in the absence of sufficient data coverage in the surrounding area, the solution for the global potential pattern would adjust itself to accommodate a greater convective flow.

\subsection{Numerical modelling of time-dependent convection}

Further to this discussion, it should be noted that using statistical averages for a quasi-steady state goes against the paradigm of a time-varying response to convection. Consider the usage of a global assimilative method in the case of data coverage only in one quadrant of the hemisphere. The statistical model used, (e.g. the APL model, Ruohoniemi and Baker, 1998), will supply averaged data corresponding to a fixed IMF orientation. This data corresponds to a quasisteady (or "equilibrium") condition, and does not consider the history of the magnetosphere-ionosphere system. By presupposing and adding in the equilibrium (final) state to a reconfiguring (intermediate) state, the global mapping procedure may artificially show a more instantaneous response. Thus caution must be exercised when interpreting data that has been "filled in" with statistical data.

Freeman (2003) described both the expanding and instantaneous responses within a single mathematical framework of the expanding-contracting polar cap (ECPC) model. His results showed that the expanding response fitted the observations better and that examining the convection patterns, the technique used by Ridley et al., made it hard to differentiate between the competing models. The expanding solution found by Freeman had the peaks of the convection pattern expanding around the polar cap boundary, but the entire equilibrium boundary was allowed to respond instantaneously. This form of expanding solution is consistent with the initial conceptual picture put forward in Cowley and Lockwood (1992), as shown in their Fig. 5. However, in later papers (Cowley and Lockwood, 1997; Lockwood and Cowley, 1999) these authors noted that the equilibrium boundary perturbation would expand tailward as newly-opened flux was added to the tail (see Figs. 2 and 3 of Lockwood and Morley, 2004). The numerical model of Lockwood and Morley (2004), hereafter Paper 1, goes beyond the Freeman (2003) model by taking these later ideas fully into account. While the high-latitude ionosphere can have a change in potential communicated across it on a timescale of tens of seconds by means of a fast Alfvén wave (Freeman et al., 1991; Ruohoniemi and Greenwald, 1998), the change in potential (associated with the development of new region 1 and region 2 field-aligned current (FAC) systems) may take time to develop.

\subsection{Two-stage ionospheric convection response}

Using magnetometer data, Murr and Hughes (2001) found that the onset of change occurred globally on timescales of about 2 min - a similar timescale to that reported by Ridley et al. (1998). However, their study also showed that the subsequent reconfiguration took place as a function of local time. The timescale for this reconfiguration was, on average, 10 min for complete reconfiguration of the ionospheric convection pattern - a result similar to those reported by Lockwood et al. and co-workers. There have been a number of other observations of a two-stage response in ionospheric convection reported in the literature. Jayachandran and McDougall (2000) used two digital ionosondes to study the polar cap convection change associated with southward turnings of the IMF and reported both a quasi-instantaneous and an expanding response. Combined observations of the polar cap using SuperDARN radars and ground magnetometers were presented by Nishitani et al. (2002) and Lu et al. (2002). These authors found that the ionospheric flow vortices responded initially within the temporal resolution of their instruments, whereas the polar cap boundary and peak magnetic perturbation responded with a delay of up to $25 \mathrm{~min}$. $\mathrm{Lu}$ et al. (2002) inferred that the two-stage response was a consequence of several interacting processes, including a fast rarefaction wave in the magnetosphere and a fast magnetosonic wave in the ionosphere. They also argued that the most important process was the feedback between the ionosphere and magnetosphere. The propagation of a fast magnetosonic wave as the cause of fast onset of change in response 
to a change in reconnection was also cited by Nishitani et al. (2002).

Lopez et al. (1999) showed in their three-dimensional MHD simulation that the convection pattern across the entire polar cap begins to change a few minutes after the arrival of the southward IMF, whereas the onset of the equatorward motion of the open-closed field line boundary depends on the local time, with equatorward motion of the midnight boundary delayed by $\sim 20 \mathrm{~min}$ relative to the onset of the boundary motion at noon. The MHD modelling study of Slinker et al. (2001) also found a fast onset of change in the ionospheric convection with a slower reconfiguration. The fast response was attributed to the propagation of a fast magnetosonic wave through the magnetosphere.

Using a numerical implementation of the CowleyLockwood paradigm, Lockwood and Morley (2004) showed that a quasi-instantaneous response could occur alongside the expected expansion, to an extent that depended on the level of pre-existing flow before the change. This paper will show how the Cowley-Lockwood (1992; 1997, hereafter CL) conceptual framework can accommodate a quasi-instantaneous response, demonstrated with the numerical model of Lockwood and Morley (2004). That the CL paradigm could accommodate this kind of response was noted in Paper 1, but was not studied in detail. In this paper we examine this effect using the Lockwood-Morley numerical model with an input reconnection rate variation having two identical pulses separated in time and making use of the techniques developed by Morley and Lockwood (2005, hereafter Paper 2).

\section{The Lockwood-Morley numerical model}

Lockwood and Morley (2004) presented a novel numerical model of the ionospheric convection response to variations in reconnection rate, incorporating all the concepts of the $\mathrm{CL}$ paradigm. The numerical model uses non-circular polar cap and equilibrium boundaries, and includes the propagation of a perturbation in the open-closed field line boundary (OCB). A full description of the model, its assumptions and limitations can be found in Paper 1. Paper 2 extended the model to calculate the ionospheric ion temperature and used the model to analyse the accuracy of the various methods used to derive expansion effects from experimental data. In this section we will outline the basic features of the model which are relevant to the expansion of the simulated flow patterns. For further details the reader is referred to Papers 1 and 2.

The model calculates the temporal evolution of the OCB and the equilibrium boundary latitudes, $\Lambda_{O C B}$ and $\Lambda_{E}$, at all magnetic local times. At any point in this temporal development of the boundaries, various outputs can be derived. A flowchart summary of the operation of the model can be seen in Fig. 1 of Paper 2.

The basic operation of the numerical model relies on three types of input: the input reconnection rate $(\varepsilon)$ variation, the initial conditions of the model high-latitude ionosphere and the constants assumed for the return of the OCB to equilibrium.

We first define the convection velocity across the boundary $\left(V^{\prime}\right)$, in its own rest frame, for a given time. The latitudinal convection velocity at the boundary $\left(V_{c n}\right)$ is then calculated and the latitudinal velocity of the OCB $\left(V_{b}\right)$ is determined. Thus the latitude of the OCB $\left(\Lambda_{O C B}\right)$ is defined at all MLT for any given simulation time, $t_{s}$. The equilibrium boundary latitude $\left(\Lambda_{E}\right)$ is then updated as a function of MLT to accommodate the new amount of open flux contained within the polar cap. The speed at which the perturbation to the equilibrium boundary propagates antisunward is the limit to the expansion of response of the OCB, and hence to the expansion of the convection pattern. The simulation time is then advanced and the input reconnection rate for the current model timestep updated. These steps then repeat for each simulation time.

The latitudinal convection velocity is used to specify the distribution of electric potential around the polar cap boundary. Using the ionospheric convection solution given in Freeman et al. (1991) and Freeman (2003) for a circular OCB, the instantaneous convection velocity for all points in the modelled high-latitude ionosphere is specified for any given timestep. Perturbation analysis is used to allow for departures of the OCB from a circular form. As in Paper 2 the ion temperature at a series of simulated stations at a constant latitude is calculated for each timestep. As there is a symmetry about the noon-midnight meridian, we employ a latitudinal ring of 72 simulated stations in the dawn hemisphere (at $67^{\circ}$; i.e. outside the polar cap), with a spacing in MLT of $10 \mathrm{~min}$. Note that the polar cap is centred on a latitude of $90^{\circ}$ in the frame used and not offset towards the nightside as in a conventional geographic or geomagnetic frame. Therefore, using a latitudinal ring in this frame ensures that the (equilibrium) OCB is equidistant from the stations at all MLTs. This allows us to isolate the azimuthal expansion of the convection pattern and avoid mixing it with any latitudinal expansion.

\section{Modelling the response to pulsed reconnection}

Paper 2 presented an examination of how using crosscorrelation methods and threshold techniques can influence the observed convection response to IMF changes in timeseries data. It was concluded that the most reliable method of recovering the expansion of the onset of the convection response is to use time series data with a threshold that is a fixed fraction of the peak response.

The first pulse of reconnection in the simulation presented in this paper is exactly identical to that used for the example of model results discussed in Paper 2. A second, identical, pulse of reconnection is added here, commencing at $t_{s}=540 \mathrm{~s}, 8 \mathrm{~min}$ after the commencement of the first pulse. The repeat period of $8 \mathrm{~min}$ is that of the mean occurrence 


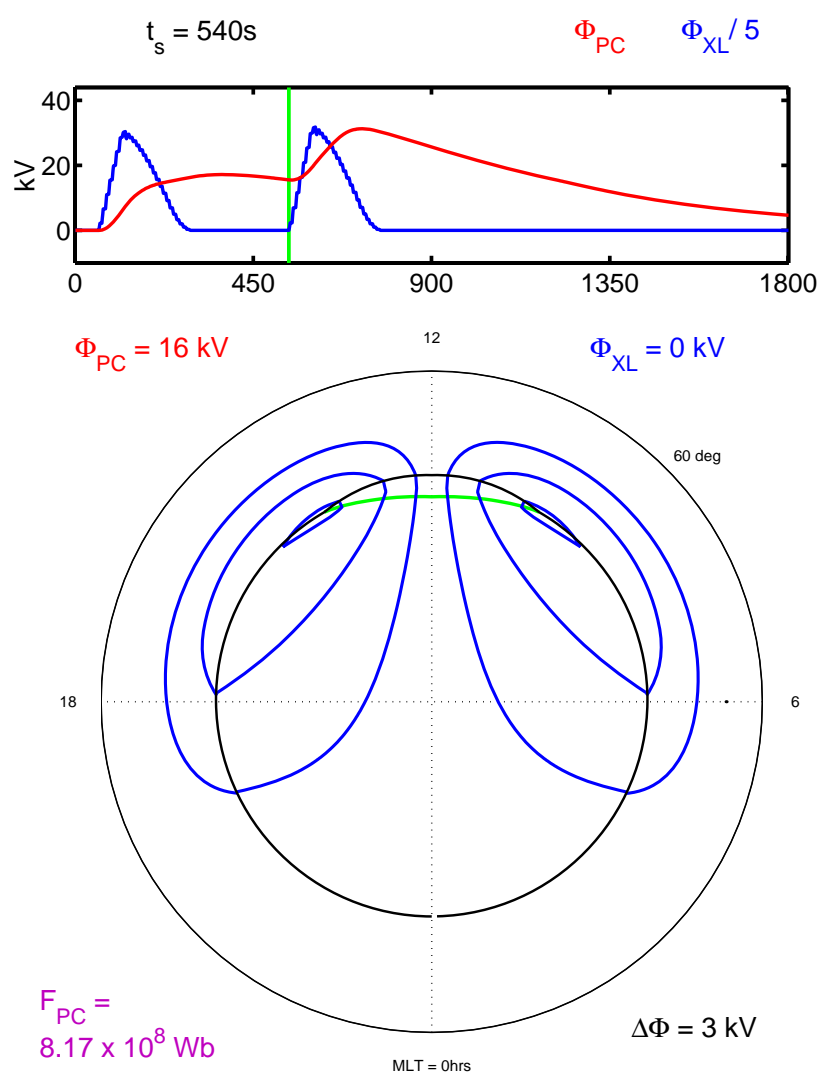

Fig. 1. A model output convection pattern for $t_{s}=540 \mathrm{~s}$, just prior to the activation of the second pulse of magnetopause reconnection. At this point the convection from the first applied pulse of reconnection has started to decay. The top panel shows the variation with simulation time, $t_{S}$, of the input reconnection voltage (in blue), $\Phi_{X L}$ divided by five to fit the same scale as the resulting transpolar voltage (in red), $\Phi_{P C}$. The vertical green line marks the simulation time of the output frame. The lower panel shows the convection pattern (using equipotentials $3 \mathrm{kV}$ apart) plotted in an MLT-magnetic latitude coordinate system. The outer circle represents the equatorward boundary of the modelled region (i.e. the region 2 FAC ring) and the inner circle represents the OCB. Non-reconnecting segments of the $\mathrm{OCB}$ are shown in black (in this case no reconnection is ongoing and so the entire OCB is black). The green line delineates the region of newly-opened flux produced by the first reconnection pulse. The open flux at this $t_{S}$ is $F_{P C}=8.17 \times 10^{8} \mathrm{~Wb}$, showing that the first pulse added $1.7 \times 10^{7} \mathrm{~Wb}$ of open flux.

period of magnetopause flux transfer events (Rijnbeek et al., 1984; Lockwood and Wild, 1993). The simulation commences with a total open flux of $F_{P C}=8 \times 10^{8} \mathrm{~Wb}$. Each reconnection pulse starts at the $\mathrm{X}$-line centre and expands towards both dusk and dawn (to the points $a$ and $b$, respectively) at a rate $\left(d|\phi X| / d t_{s}\right)$ which we set to $0.167^{\circ} \mathrm{s}^{-1}$, corresponding to $1 \mathrm{~h}$ of MLT per $1.5 \mathrm{~min}$. Similarly, the end of each reconnection pulse is first seen at 12:00 MLT, $t=1 \mathrm{~min}$ after the onset, and propagates towards both $a$ and $b$ at the
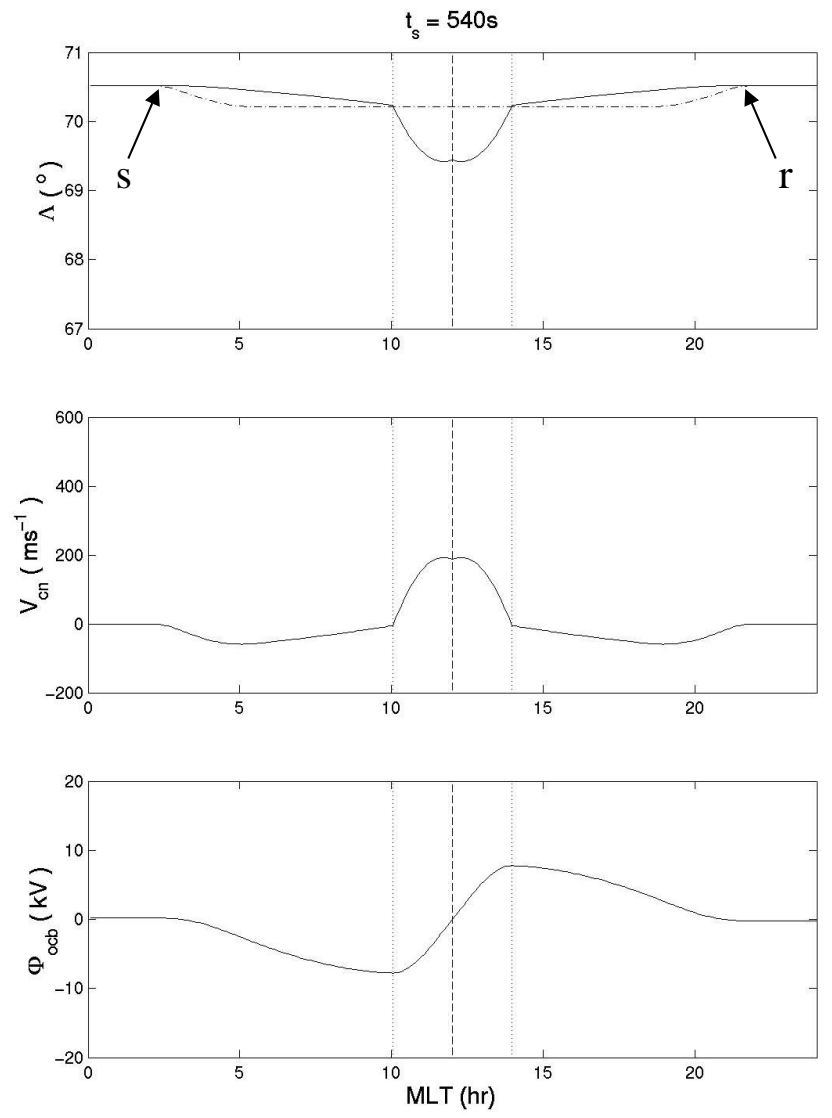

Fig. 2. Model output boundary characteristics corresponding to Fig. 1. The top panel shows the latitude as a function of MLT, of the OCB (solid line), $\Lambda_{0}$, and the equilibrium boundary (dashdot line), $\Lambda_{E}$. The middle panel shows the poleward convection velocity at the OCB, $V_{c n}$, caused by the motion of the boundary. The bottom panel shows the electrostatic potential around the OCB, $\Phi_{O C B}$. The vertical dashed line in each panel marks the centre of the merging gap, at 12:00 MLT, and the dotted lines mark the maximum extent of the merging gap. These lie at 10:00 and 14:00 MLT. Note that the perturbation to the equilibrium boundary has propagated to the points marked $s$ and $r$.

same rate. Thus each pulse causes the reconnection to be active at any MLT between $a$ and $b$ for $1 \mathrm{~min}$. Two such pulses are included in the present simulation, with onsets at $t_{s}=1 \mathrm{~min}$ and $t_{s}=9 \mathrm{~min}$. The background reconnection rate outside the two pulses is taken to be zero.

A constant reconnection electric field of $0.2 \mathrm{~V} \mathrm{~m}^{-1}$ is applied at each MLT of the ionospheric projection of the X-line for 1 minute, expanding from noon MLT to cover a maximum X-line extent of $4 \mathrm{~h}$ of MLT. This azimuthal movement of the active reconnection region is as proposed and deduced from observations by, e.g., Lockwood et al. (1993a), Milan et al. (2000) and McWilliams et al. (2001). The model response is identical to that presented in Paper 2 until the commencement of the second pulse. The effects of an existing flow-pattern (that is, a disturbed OCB) caused by the first 


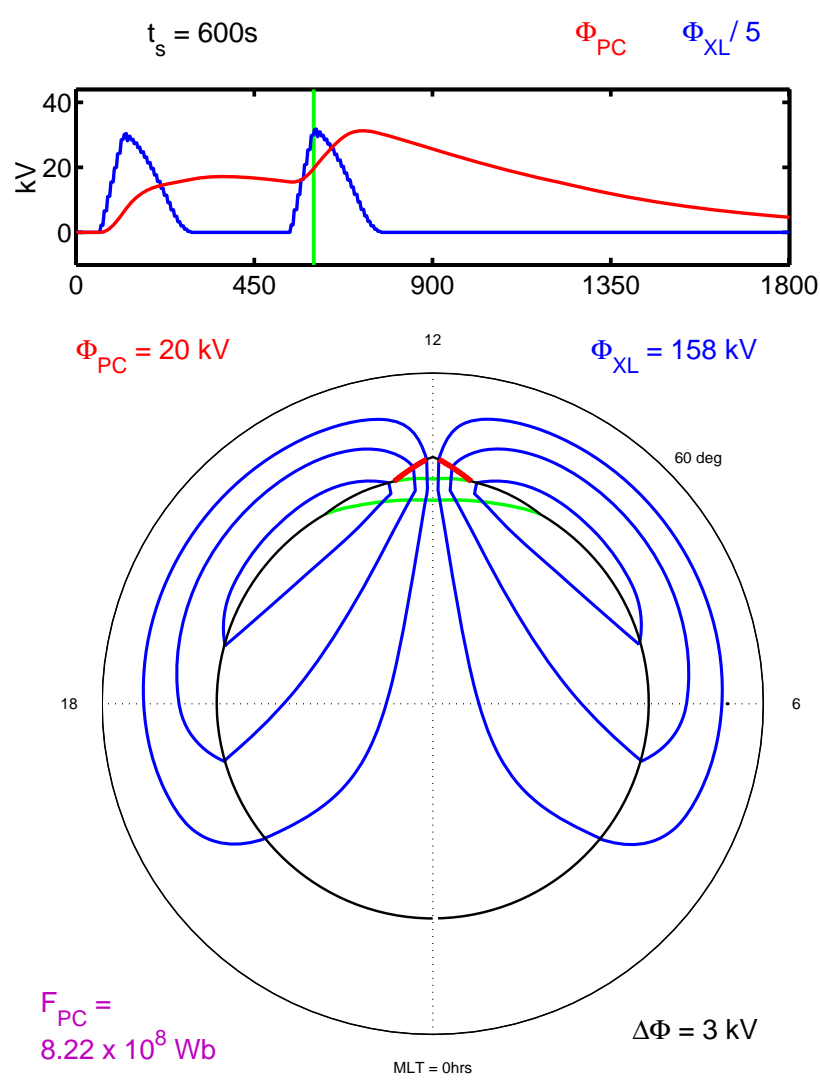

Fig. 3. A model output convection pattern for $t_{s}=600 \mathrm{~s}, 60 \mathrm{~s}$ after the commencement of the second pulse of reconnection. The format is the same as Fig. 1. Active segments of the OCB, which map to the magnetopause $\mathrm{X}$-line where reconnection is ongoing, are shown in red.

pulse on the model response to the second pulse are discussed below.

Prior to the onset of the second pulse of reconnection, the flows generated by the initial pulse of reconnection have not yet subsided and the convection pattern at simulation time $t_{s}=540 \mathrm{~s}$ is shown in Fig. 1. A full sequence of flow pattern plots for a similar reconnection scenario to that employed here is given in Paper 1.

At this time the second pulse of reconnection is about to commence. The convection pattern is diminishing in strength while the extent of the pattern continues to grow over the polar region. The upper panel of Fig. 1 shows the variation with simulation time, $t_{s}$, of the input reconnection voltage, $\Phi_{X L}$ (in blue), divided by five. It can be seen that the reconnection pulses are spaced $8 \mathrm{~min}$ apart and that they are identical. The polar cap voltage, $\Phi_{P C}$ (in red), has already peaked at about $18 \mathrm{kV}$ by this time and has subsequently decayed slightly - this is shown in the presence of only 3 plotted equipotentials, which are separated by $3 \mathrm{kV}$ and centred about zero. The boundary locations for this time are shown
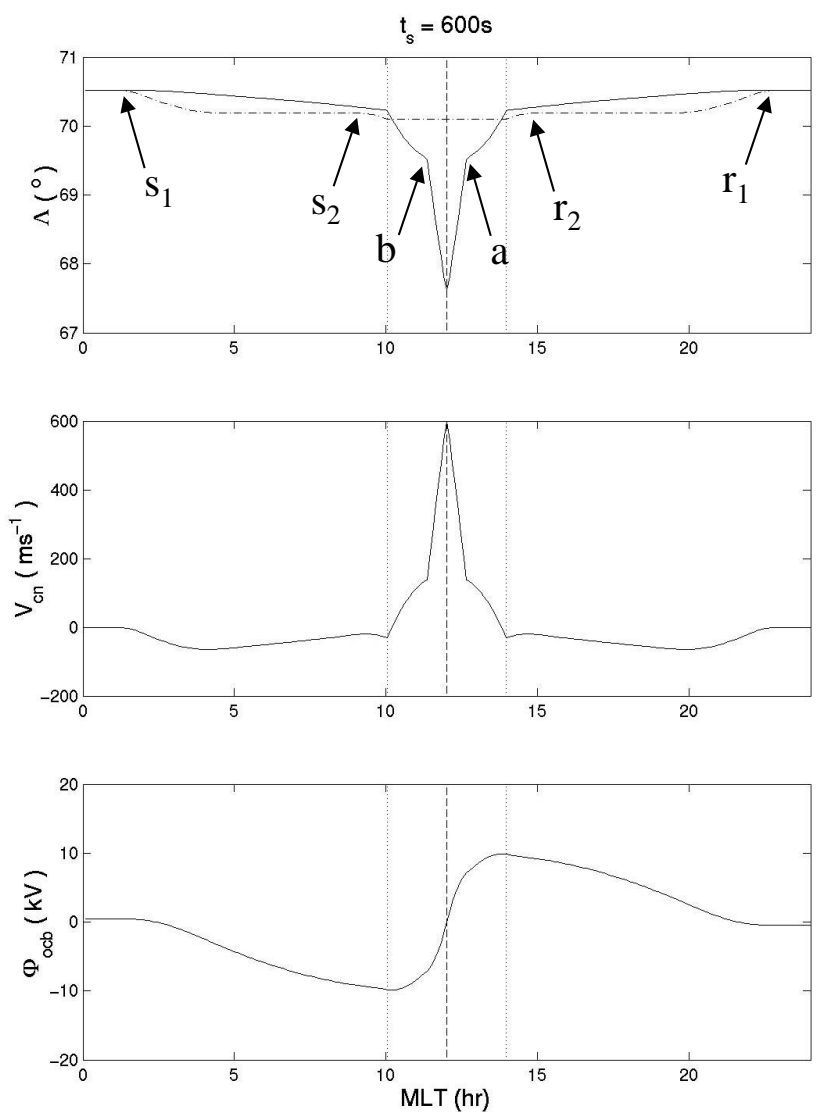

Fig. 4. Model output boundary characteristics corresponding to Fig. 3. The format is the same as Fig. 2. $r_{1}$ and $s_{1}$ mark the extent of the perturbation to the equilibrium boundary (dash-dot line) caused by the first pulse of reconnection. Similarly, $r_{2}$ and $s_{2}$ mark the extent of the perturbation to the equilibrium boundary due to the second reconnection pulse. The extent of the active OCB (the active "merging gap", mapping to the segment of the magnetopause where reconnection is ongoing - solid line) is between the points $a$ and $b$. The middle and lower panels show the poleward convection velocity at the OCB and the electrostatic potential around the OCB, respectively.

in Fig. 2 (top panel), along with the convection velocity in the boundary rest frame (middle panel) and the electrostatic potential along the OCB (lower panel).

The equilibrium boundary perturbation - the point at which the OCB can have responded to the added open flux has propagated beyond 03:00 and 21:00 MLT (points $s$ and $r$ ) on the dawn and dusk sides respectively. The poleward flow between 10:00 and 14:00 MLT (the maximum extent of the merging gap) is balanced by the equatorward flow outside of this region.

Figure 3 shows the convection pattern at $t_{s}=600 \mathrm{~s}$. This shows the model output $60 \mathrm{~s}$ after the initiation of the second pulse of reconnection. The actively reconnecting segments of the merging gap are shown by the thick red lines. The green lines delineate the regions of newly-opened flux formed by 


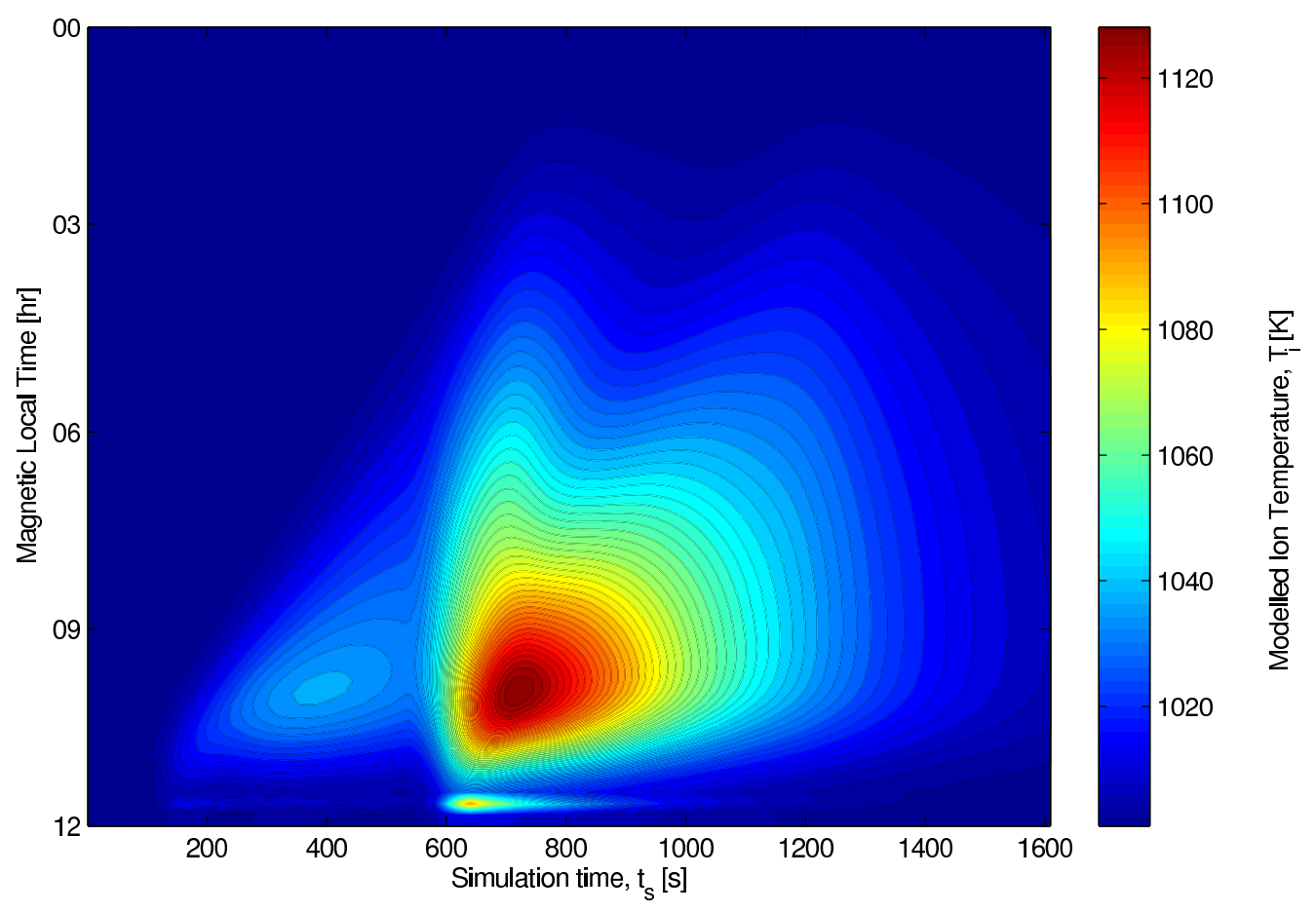

Fig. 5. A formedogram of the amplitude of the ion temperature response to the series of two reconnection pulses. The plot colour-codes the ion temperature as a function of simulation time $t_{s}$ (horizontal axis) and magnetic local time (vertical axis: where 00 MLT corresponds to simulated station number 72 and 12:00 MLT corresponds to simulated station 0; all stations are at a latitude of $67^{\circ}$ ). This plot is the same as Fig. 6 of Morley and Lockwood (2005), but with a second burst of reconnection, identical to the first, 8 min after the commencement of the first. Note the change in the ion temperature scale compared with that used by Morley and Lockwood (2005), which is required because much higher temperatures are driven in response to the second pulse.

each reconnection pulse. The ionospheric merging gap voltage is at its peak of $\Phi_{X L}=158 \mathrm{kV}$ and the transpolar voltage, $\Phi_{P C}$, has risen from $16 \mathrm{kV}$ to $20 \mathrm{kV}$ (but does not reach its peak value of near $30 \mathrm{kV}$ until $150 \mathrm{~s}$ later).

Figure 4 shows the boundary characteristics at $t_{s}=600 \mathrm{~s}$ in the same format as Fig. 2. The latitudes of the OCB (solid line) and equilibrium boundary (dash-dot line), as a function of MLT, are shown in the upper panel. A boundary erosion identical to that caused by the first pulse is superimposed on the already distorted boundary. This new bulge is bounded by points $a$ and $b$. The extent of the equilibrium boundary perturbation to the second pulse is marked by points $r_{2}$ and $s_{2}$, while the corresponding limits for the perturbation due to the first pulse, $r_{1}$ and $s_{1}$, have expanded to nearer midnight compared with Fig. 2. The middle panel shows $V_{c n}$ has increased by a substantial amount between $a$ and $b$. This increase is not balanced (as it was at the same stage for the first pulse) by equatorward flow between both $a$ and $r_{2}$ and $b$ and $s_{2}$. Comparison of the lower panel with Fig. 2 shows that the potential around the OCB is enhanced everywhere between $r_{1}$ and $s_{1}$.

Figure 5 shows the formedogram (from the Greek formedon - meaning "in layers crosswise") for the ion temperature enhancement resulting from this two-pulse reconnection variation. This format show the contours of the ion temperature enhancement, resulting from the enhanced ion flow, as a function of simulation time, $t_{s}$, and magnetic local time, where 12:00 MLT corresponds to station 0 . The simulated stations are at a latitude of $67^{\circ}$ and are positioned around the dawn hemisphere with a spacing in MLT of $10 \mathrm{~min}$. We have examined the effect of the initial pulse of reconnection in isolation in Paper 2. This first pulse of reconnection produces convection in the model, as the OCB responds to displacement from its equilibrium configuration. When the second pulse of reconnection is applied, the convection resulting from the first pulse is still present. Although the response in ion heating to the first pulse is identical to that presented in Paper 2, comparison of Fig. 5 with Fig. 7 of Paper 2 highlights the difference in the level of the response to the second pulse. This is also apparent from Fig. 5 alone when one compares the initial response to reconnection pulses 1 and 2, the maximum simulated ion temperature reaching over $1120 \mathrm{~K}$. This is significantly larger than the peak of about $1040 \mathrm{~K}$ in response to the first reconnection pulse. The temperature anomaly in the data series for simulated station $3(\simeq 11: 30 \mathrm{MLT})$ is not an erroneous point: 


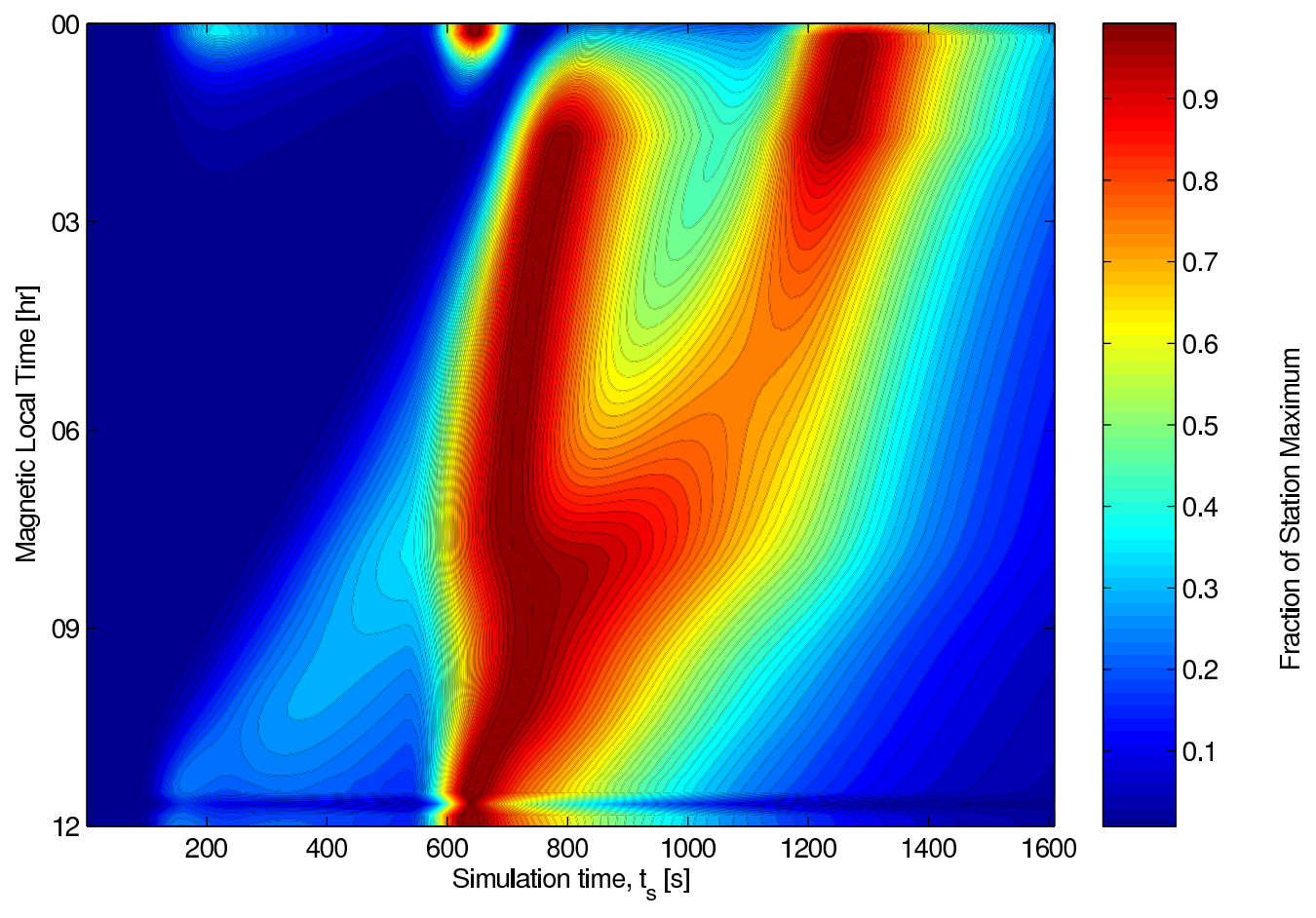

Fig. 6. Same as Fig.5, but normalized to the maximum response at each simulated station. Contours of $\left(\mathrm{T}_{i}-\mathrm{T}_{i 0}\right) /\left(\mathrm{T}_{p}-\mathrm{T}_{i 0}\right)$ are plotted as a function of MLT and simulation time, $t_{s}$, where $\mathrm{T}_{i 0}$ is the value of $\mathrm{T}_{i}$ at $t_{s}=0$ and $\mathrm{T}_{i p}$ is the peak temperature seen at that station during the simulation.

it arises from the proximity of the active reconnection X-line footprint and was discussed in Paper 2. This is the only station to be engulfed by the temperature enhancement that is localized around the end of the merging gap.

Figure 6 shows the ion heating response (as a function of both simulation time, $t_{s}$, and MLT) by presenting the ion temperature change for each MLT, normalized to the local peak change at that MLT. For the first pulse, the response is weaker at all MLT and shows a clear anti-sunward propagation, with the enhancement being later at larger virtual station number (i.e. at MLT increasingly removed from noon). However, the (larger) response to the second reconnection pulse shows both a quasi-instantaneous response and an expanding response. The character of the response varies with MLT. The apparently instantaneous response present near midnight is due to numerical noise (of order $0.1 \mathrm{~K}$ ) being amplified by the normalization as described in Paper 2. Visual comparison with Fig. 5 shows that the amplitude of the temperature change is negligible.

The two reconnection pulses are identical and the difference in response to the two highlighted by Figs. 5 and 6 reveals that the effect of a pre-existing background flow is substantial. Equivalently, localized addition of open flux to a non-equilibrium polar cap can cause a large-scale response. This will be explained in the following section.

\section{Discussion}

We have presented results from the Lockwood and Morley (2004) numerical model predicting the effect of superposing a reconnection pulse on both an undisturbed and an alreadydisturbed ionosphere. The inputs to the model are identical to those presented in Paper 2, with the exception of the input reconnection rate which now includes a second, identical, pulse of reconnection commencing at $t_{s}=540 \mathrm{~s}, 8 \mathrm{~min}$ after the commencement of the first reconnection pulse.

The convection patterns presented in Figs. 1 and 3 (for $t_{s}=540 \mathrm{~s}$ and $600 \mathrm{~s}$, respectively) appear to show a global increase in convection strength in response to the second of the two applied reconnection pulses. For the first pulse (perturbing an undisturbed MI system) the effect is initially localised around the reconnection site, but then expands. The second pulse (perturbing an MI system that has already been perturbed by the first pulse) has a larger response which has both instantaneous and expanding characteristics (as revealed by Fig. 6). The principles of this effect can be understood from Fig. 4. The top panel shows the latitudes of both the OCB, $\Lambda_{O C B}$, and the equilibrium boundary, $\Lambda_{E}$, as functions of MLT. The perturbation to the equilibrium boundary caused by the first reconnection pulse has almost reached 23:00 MLT on the dusk side $\left(r_{1}\right)$ and near 01:00 MLT on the dawn side $\left(s_{1}\right)$. Thus the convection pattern is still expanding in 
response to the first pulse of reconnection. This perturbation due to the first pulse is close to encircling the polar cap, allowing the entire OCB to respond, and thus in the absence of the second pulse the convection pattern would have decayed exponentially in a quasi shape-preserving manner (see Paper 2). However, the perturbation to the equilibrium boundary caused by the addition of new open flux to the polar cap is initially confined to the reconnection site with a subsequent expansion at a velocity $d \phi_{r 2} / d t_{s}$, with point $r_{2}$ propagating eastward away from $a$ and $s_{2}$ propagating westward away from $b$. This is exactly the same perturbation motion experienced by the equilibrium boundary in response to the first pulse of reconnection.

The residual effect of the first pulse can be seen in the OCB latitude by the fact that it has migrated equatorward at MLTs outside the merging gap (except near midnight, where neither $\Lambda_{E}$ nor $\Lambda_{O C B}$ have yet been influenced). However, the OCB is still poleward of its equilibrium position everywhere outside the ionospheric footprint of the X-line (the "merging gap"). The effect of the second pulse can be seen as a large equatorward migration within the active merging gap $a b$. The effects of the two pulses can be also be distinguished in the poleward convection speeds, with equatorward motion $\left(V_{c n}<0\right)$ at MLTs outside the merging gap (except near midnight) and strong poleward flow $\left(V_{c n}>0\right)$ inside the merging gap. An enhancement of the equatorward flow is seen just outside of the merging gap (between $a$ and $r_{2}$ and between $b$ and $s_{2}$ ), but this is much weaker than the poleward flow between $a$ and $b$. This should be contrasted with the situation for the equivalent time following the first pulse (Fig. 4 in Paper 1) where the total equatorward flow (between $a$ and $s_{1}$ and between $b$ and $r_{1}$ ) matches the total poleward flow between $a$ and $b$ : this is the case for all times when a zero-flow equilibrium system is perturbed by a single pulse of reconnection and can also be seen in Fig. 2. Indeed, in the bottom panels of Figs. 2 and 4 it can be seen that the additional equatorward flow between $a$ and $s_{2}$ and between $b$ and $r_{2}$ causes only a very small perturbation to the potential distribution around the OCB.

In the CL model the displacement from equilibrium is what determines the flow normal to the OCB at a given location. Where the OCB is close to the equilibrium boundary, only weak flow will be excited. In this example the OCB and equilibrium boundary are close together at $s_{2}$ and $r_{2}$, hence $\left|\Lambda_{O C B}-\Lambda_{E}\right|$ is small and $V_{c n}$ is near zero. This situation arises because the equilibrium boundary at these MLTs is perturbed equatorward by the second pulse of reconnection. However, the OCB has been eroded equatorward by a similar amount in response to the first pulse. In the absence of the perturbation due to the first pulse the streamlines would return across the OCB sunward of $s_{2}$ and $r_{2}$. Near these points at this simulation time, $t_{s}=600 \mathrm{~s}$, the flow that would have been caused by the first pulse in isolation is poleward and comparable in magnitude to the equatorward flow that would result from the second pulse in isolation. They can thus be considered to cancel each other out here, and the equatorward flow required because the ionosphere is incompressible must take place elsewhere (between $r_{1}$ and $r_{2}$ and between $s_{1}$ and $s_{2}$, i.e. closer to midnight). The flow increases at all MLTs between $s_{1}$ and $r_{1}$. It is important to note that the flows do not superpose - rather it is the boundary perturbations that superpose and their subsequent return towards their equilibrium position governs how the flow responds.

This "global" strengthening of the convection pattern may provide an explanation for the quasi-simultaneous response reported by several authors (e.g. Ridley et al., 1997; Ruohoniemi and Baker, 1998). In this context, it is useful to note that Ridley et al. studied perturbations to the flow in response to southward turnings of the IMF using "residual potential patterns". These were obtained by subtracting from each observed potential a pre-existing potential from before the IMF change. Thus in their examples there was pre-existing flow as in the simulation presented here.

\subsection{Ion temperature response}

As described in Paper 2, the convection response is also clearly seen in the ion heating - the dependence of ion heating on the convection velocity approximately follows a squared relationship (we here neglect the effects of thermospheric neutral winds and their (slow) response to the convection change). The formedogram of simulated ion temperature (as a function of simulation time and MLT) shown in Fig. 5 reveals response to the first pulse of reconnection which, as in Paper 2, expands away from noon at about $11 \mathrm{~km} \mathrm{~s}^{-1}$. The second pulse of reconnection commences at $t_{s}=540 \mathrm{~s}$. The erosion of the OCB is almost identical to that caused by the first pulse of reconnection applied. However, the response in the ion heating reveals a very different convection response. The response in frictional ion heating, hence in the convection, appears in Fig. 5 to be nearly simultaneous across the entire dayside. However, as discussed earlier, observing at a fixed threshold level can lead to a significantly reduced apparent expansion velocity and to a reduced apparent extent of the expansion. This formedogram also shows a bifurcation in the response to the second pulse, with an apparent expanding component in addition to the more instantaneous response.

Figure 6 shows the ion heating response (as a function of simulation time and MLT), where the data from each simulated station has been normalized to the local maximum -i.e. the contours represent the fraction of peak change in ion temperature at a given MLT. Here the effect of the reconnection onset just after $t_{s}=540 \mathrm{~s}$ is clearly seen globally, with a rise to peak convection response within $3 \mathrm{~min}$ of the commencement of addition of open flux by the second pulse of reconnection. Only on the nightside is there any evidence for an antisunward expansion of this quasi-instantaneous response. This interaction between the flow present prior to the second pulse and the new flow, giving the global response, then falls away and a second, expanding, response can be seen moving 


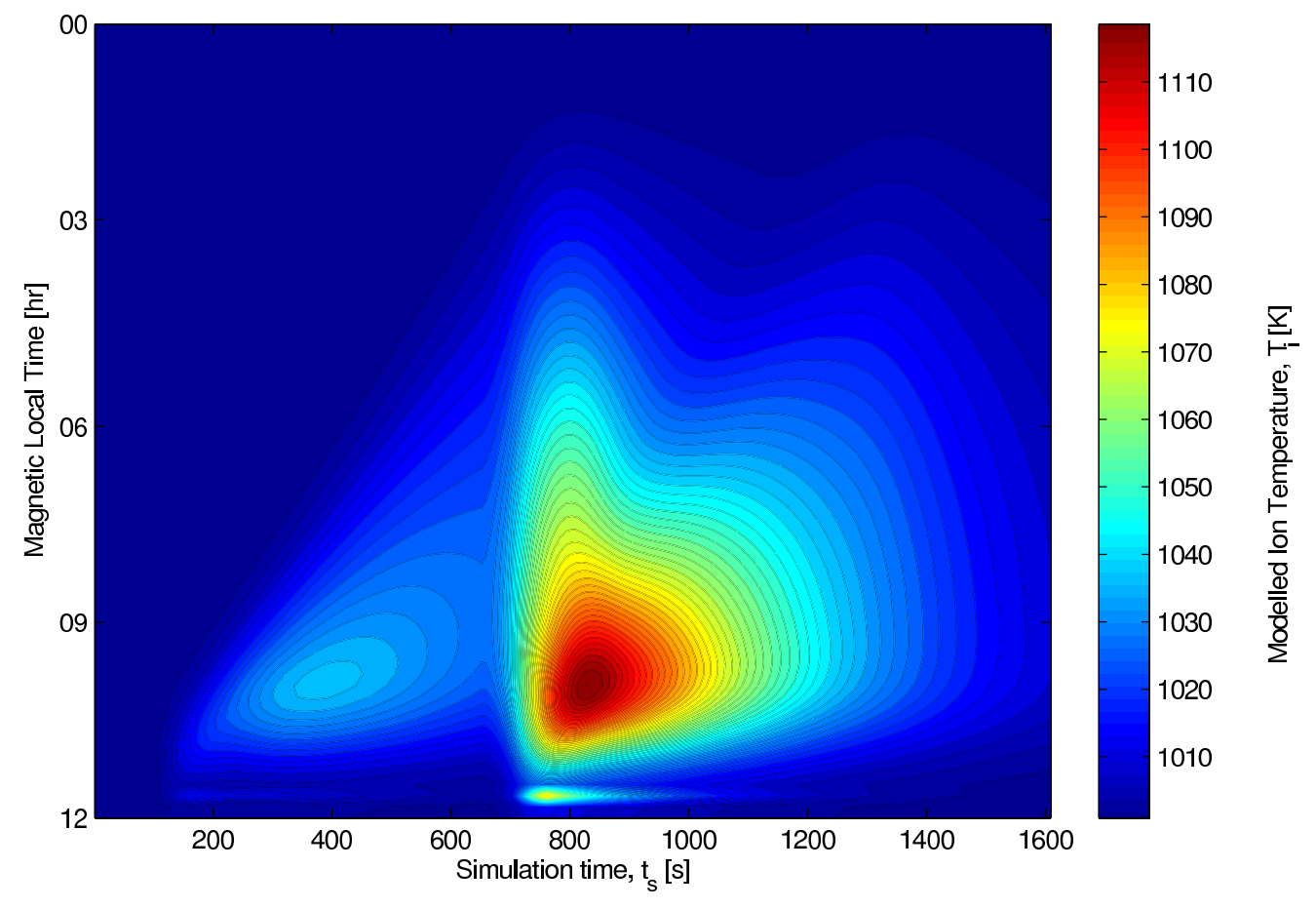

Fig. 7. Same as Fig.5, but for a reconnection pulse separation of $10 \mathrm{~min}$. Note the change in the ion temperature scale used compared to Fig. 5 because lower temperatures are driven by the second pulse; this results from the greater separation between pulses allowing the remnant flow to decay further.

anti-sunward with a similar phase velocity to that for the first pulse, i.e. with a velocity of about $11 \mathrm{~km} \mathrm{~s}^{-1}$. The peak ion heating response at 00:00 MLT is seen to occur at $t_{s}=1250 \mathrm{~s}$, about 12 min after the onset of the second reconnection pulse, corresponding to the timescale for reconfiguration of the entire convection pattern. The temporal separation between the two responses becomes sufficiently large that we can separate them as we approach the dawn-dusk meridian. It is interesting to note that in this case the expanding response is of lesser magnitude than the quasi-instantaneous response, as measured by the ion heating, falling as low as $70 \%$ of the peak response at some MLTs.

\subsection{The effect of pulse separation}

To illustrate the effect of the separation between reconnection pulses on the quasi-instantaneous and expanding responses we here use the input reconnection scenario described earlier in this paper, but increase the separation between the applied pulses of reconnection to $10 \mathrm{~min}$; i.e. the second pulse of reconnection now commences at $t_{s}=660 \mathrm{~s}$. The formedograms of simulated ion temperature for this case are shown in Figs. 7 and 8 (corresponding to Figs. 5 and 6 , respectively).

Comparison of Fig. 7 with Fig. 5 shows that the form of the response in ion temperature is qualitatively similar. Since the reconnection pulses have a greater separation in time, the flow excited by the initial pulse of reconnection has decayed further at the onset of the second pulse. The response to the second pulse is therefore slightly lower in magnitude than in the previous example.

Figure 8 shows the effect of the increased pulse separation on the heating, as a fraction of the maximum response at each station. As before, the quasi-instantaneous response is present across much of the high-latitude ionosphere. Here the onset of the ion heating response occurs over a greater MLT extent than before, and no longer shows the antisunward expansion of the quasi-instantaneous response present in Fig. 6. This global strengthening of the ionospheric convection again shows the greatest perturbation in the temperature profiles for each simulated station. This is explained by the fact that the expanding response, which is smaller in this example because of the greater pulse separation, weakens as it spreads around the auroral oval towards the nightside. It therefore has less effect on the magnitude of the change in convection, and the resultant ion heating. This can be seen in the different ion temperatures just after the onset of the second reconnection pulse. For the case of an 8-min separation the peak temperature measured here is $1125 \mathrm{~K}$, whereas for a pulse separation of $10 \mathrm{~min}$ the peak temperature around $10 \mathrm{~K}$ lower. However, the heating associated with the quasi-instantaneous response covers a larger MLT extent. The decay of the instantaneous response is with the 


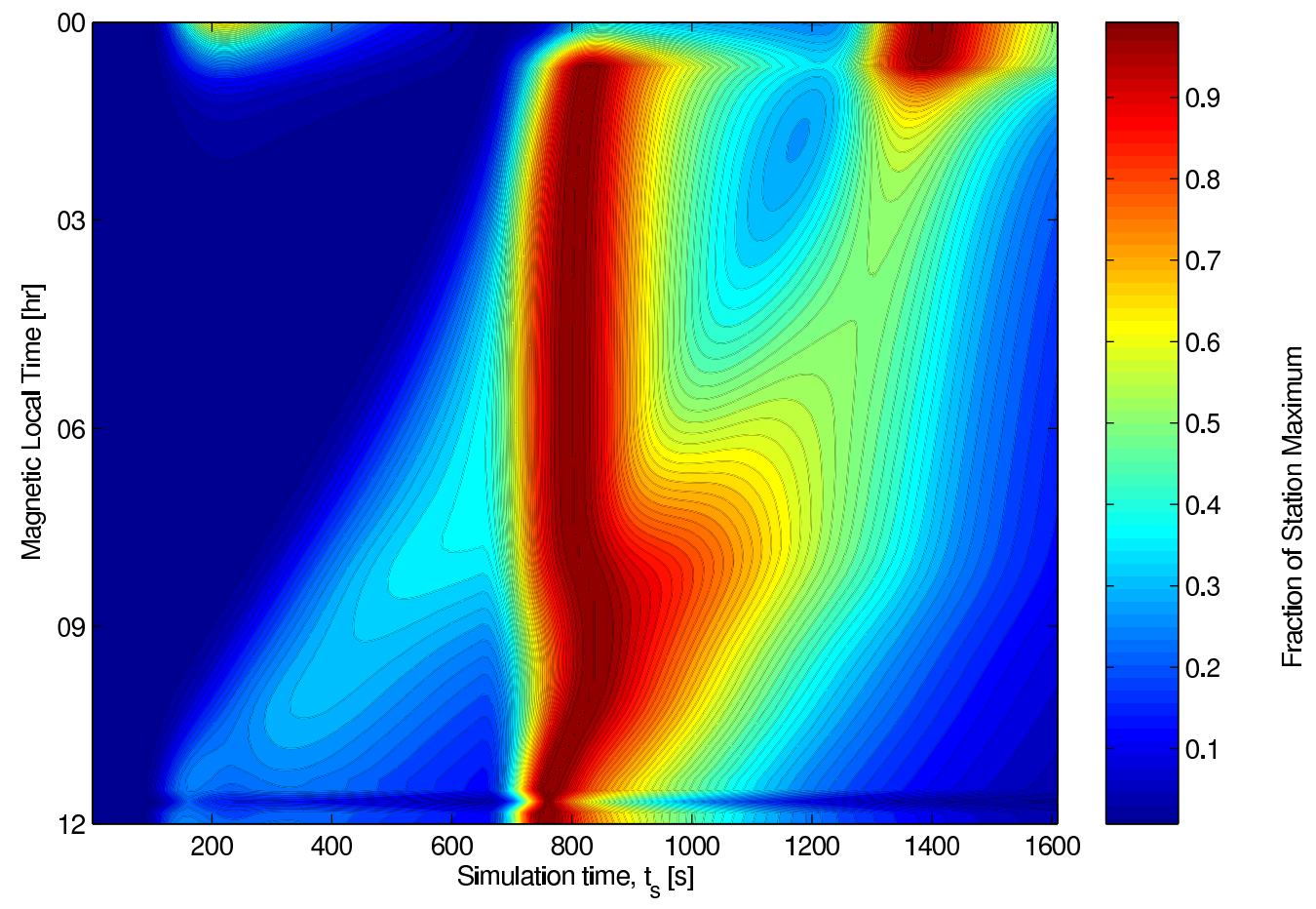

Fig. 8. Same as Fig.6, but for a reconnection pulse separation of $10 \mathrm{~min}$.

same time constant as that for the decay of the residual flow associated with the first pulse (of order $10 \mathrm{~min}$ ).

The superposed and expanding responses are still difficult to distinguish on the dayside, where the simulated ion temperature increase is greatest. However, on the dayside the response to an applied pulse of reconnection is observed in the ion heating as a temperature enhancement expanding away from the reconnection footprint. In these examples the quasi-instantaneous strengthening of the convection pattern, as seen in the associated ion heating, can only be fully separated from the classic Cowley-Lockwood $(1992 ; 1997)$ expanding response on the nightside.

For small pulse separations, the instantaneous response will not be seen as the two reconnection pulses effectively merge into a single, longer-lived pulse. Comparison of Figs. 5 and 7 illustrates how the magnitude of the instantaneous response in ion heating decays with increasing pulse separations above $8 \mathrm{~min}$, eventually disappearing to leave two identical expanding responses of the type presented in Paper 2.

\section{Conclusions}

We have shown that both expanding (on a $\sim 12$-min timescale) and quasi-instantaneous responses of the ionospheric convection to magnetopause reconnection can be explained with the Cowley and Lockwood $(1992,1997)$ model of flow excitation in the coupled magnetosphere-ionosphere
(MI) system. This model has a key feature of timedependence, necessarily considering the prior history of the MI region. The work presented in this paper shows that a residual flow from magnetopause reconnection can produce a quasi-instantaneous global ionospheric convection response; residual flow may also be present from tail reconnection, which will superpose constructively to give a similar effect. The flow generated by the second pulse is not a simple superposition of the flow patterns associated with the two pulses in isolation. Rather, it is the boundary perturbations (the OCB and equilibrium boundary) which are superposed and the combined effect of the two pulses gives a quite different response to the isolated patterns or the superposition of the two as the OCB migrates back towards its equilibrium position.

As the OCB will frequently be in motion from such prior reconnection (both at the dayside magnetopause and at a magnetotail neutral line), it is expected that there will usually be some level of combined response to dayside reconnection. IMF $B_{y}$ and its variations will also have an effect, though modelling of the effect of a strong $B_{y}$ component cannot be achieved with the current model, since symmetry is assumed in the analytic solution of Laplace's equation.

The example presented offers a plausible explanation for reported global responses within the Cowley-Lockwood paradigm. The model predicts a quasi-instantaneous strengthening of the convection pattern along with the expected expanding reconfiguration response, in accordance 
with observations by, for example, Murr and Hughes (2001) and $\mathrm{Lu}$ et al. (2002). Note that these responses in our modelling are not due to the presence of different magnetosonic wave speeds. Other mechanisms have been proposed to explain certain cases, including an overdraped lobe causing reconnection onset simultaneously across a large MLT extent (e.g. Shepherd et al., 1999). However, our results show that these are also not necessary to explain the observations.

In the sample results presented, the "global" and "expanding" responses in the bulk flow occurred too close together to be separated across much of the dayside. The reconfiguration of the ionospheric flows in response to the applied pulse of reconnection is seen to occur as an expanding reconfiguration of the type discussed by Cowley and Lockwood (1992). Further, the separation between dayside reconnection events, is seen to be an important factor in the degree of separation between the global and expanding responses, and in the level of the quasi-instantaneous response caused by the superposition of the OCB and equilibrium boundary perturbations. In the present paper we have concentrated on the effects of pulses in the magnetopause reconnection rate; however, similar effects will also be produced by pulses in the nightside reconnection in the cross-tail current sheet.

Acknowledgements. This work was supported by the UK Particle Physics and Astronomy Research Council and the Cooperative Research Centre for Satellite Systems through the Commonwealth of Australia CRC Program.

Topical Editor M. Pinnock thanks K. McWilliams and S. Milan for their help in evaluating this paper.

\section{References}

Cowley, S. W. H. and Lockwood, M.: Excitation and decay of solar-wind driven flows in the magnetosphere-ionosphere system, Ann. Geophys., 10, 103-115, 1992.

Cowley, S. W. H. and Lockwood, M.: Incoherent scatter radar observations related to magnetospheric dynamics, Adv. Space Res., 20 (4/5), 873-882, 1997.

Etemadi, A., Cowley, S. W. H., Lockwood, M., Bromage, B. J. I., Willis, D. M., and Lühr, H.: The dependence of high-latitude flows on the North-South component of the IMF: A high time resolution correlation analysis using EISCAT "Polar" and AMPTE UKS and IRM data, Planet. Space Sci., 36, 471-498, 1988.

Freeman, M. P.: A unified model of the response of ionospheric convection to changes in the interplanetary magnetic field, J. Geophys. Res., 108(A1), 1024, doi:10.1029/2002JA009385, 2003.

Freeman, M. P. and Southwood, D. J.: The effect of magnetospheric erosion on mid- and high-latitude ionospheric flows, Planet. Space Sci., 36, 509-522, 1988.

Freeman, M. P., Ruohoniemi, J. M., and Greenwald, R. A.: The determination of time-stationary two-dimensional convection patterns with single-station radar, J. Geophys. Res., 96, 15735$15749,1991$.
Jayachandran, P. T. and McDougall, J. W.: Central polar cap convection response to short duration southward Interplanetary Magnetic Field, Ann. Geophys., 18, 887-896, 2000.

Khan, H. and Cowley, S. W. H.: Observations of the response time of high-latitude ionospheric convection to variations in the interplanetary magnetic field using EISCAT and IMP-8 data, Ann. Geophys., 17, 1306-1335, 1999.

Lockwood, M. and Cowley, S. W. H.: Comment on "A statistical study of the ionospheric convection response to changing interplanetary magnetic field conditions using the assimilative mapping of ionospheric electrodynamics technique" by A. J. Ridley et al., J. Geophys. Res., 104, 4387-4391, 1999.

Lockwood, M. and Morley, S. K.: A numerical model of the ionospheric signatures of time-varying magnetic reconnection: I. Ionospheric convection, Ann. Geophysicae, 22, 73-91, 2004.

Lockwood, M. and Wild, M. N.: On the quasi-periodic nature of magnetopause flux transfer events, J. Geophys. Res., 98, 59355940, 1993.

Lockwood, M., van Eyken, A. P., Bromage, B. J. I., Willis, D. M., and Cowley, S. W. H.: Eastward propagation of a plasma convection enhancement following a southward turning of the interplanetary magnetic field, Geophys. Res. Lett., 13, 72-75, 1986.

Lockwood, M., Denig, W. F., Farmer, A. D., Davda, V. N., Cowley, S. W. H., and Lühr, H.: Ionospheric signatures of pulsed reconnection at the Earth's magnetopause, Nature, 361, 424-427, 1993a.

Lopez, R. E., Wiltberger, M., Lyon, J. G., Goodrich, C. C., and Papadopoulos, K.: MHD simulations of the response of highlatitude potential patterns and polar cap boundaries to sudden southward turnings of the interplanetary magnetic field,, Geophys. Res. Lett., 26, 967-970, 1999.

Lu, G., Holzer, T. E., Lummerzheim, D., Ruohomiemi, J. M., Stauning, P., Troshichev, O., Newell, P. T., Brittnacher, M., and Parks, G.: Ionospheric response to the interplanetary magnetic field southward turning: Fast onset and slow reconfiguration, J. Geophys. Res., 107, 1153, doi:10.1029/2001JA000324, 2002.

McWilliams, K. A., Yeoman, T. K., Sigwarth, J. B., Frank, L. A., and Brittnacher, M.: The dayside ultraviolet aurora and convection responses to a southward turning of the interplanetary magnetic field, Ann. Geophys., 19, 707-722, 2001.

Milan, S. E., Lester, M., Cowley, S. W. H., and Brittnacher, M.: Convection and auroral response to a southward turning of the IMF: Polar UVI, CUTLASS, and IMAGE signatures of transient magnetic flux transfer at the magnetopause, J. Geophys. Res., 105, 15 741-15 755, 2000.

Morley, S. K. and Lockwood, M.: A numerical model of the ionospheric signatures of time-varying magnetic reconnection: II Measuring expansions in the ionospheric flow response, Ann. Geophys., 23, 2501-2510, 2005.

Murr, D. L. and Hughes, W. J.: Reconfiguration timescales of ionospheric convection, Geophys. Res. Lett., 28, 2145-2148, 2001.

Nishitani, N., Ogawa, T., Sato, N., Yamagishi, H., Pinnock, M., Villain, J. P., Sofko, G., and Troshichev, O.: A study of the dusk convection cells response to an IMF southward turning, J. Geophys. Res., 107, 1036, doi:10.1029/2001JA900095, 2002.

Richmond, A. D. and Kamide, Y.: Mapping electrodynamic features of the high-latitude ionosphere from localized observations: techniques, J. Geophys. Res., 93, 5741-5759, 1988. 
Ridley, A. J., Lu, G., Clauer, C. R., and Papitashvilli, V. O.: Ionospheric convection during nonsteady interplanetary magnetic field conditions, J. Geophys. Res., 102, 14 563-14579, 1997.

Ridley, A. J., Lu, G., Clauer, C. R., and Papitashvilli, V. O.: A statistical study of the ionospheric convection response to changing interplanetary magnetic field conditions using the assimilative mapping of ionospheric electrodynamics technique, J. Geophys. Res., 103, 4023-4039, 1998.

Rijnbeek, R. P., Cowley, S. W. H., Southwood, D. J., and Russell, C. T.: A survey of dayside flux transfer events observed by ISEE 1 and 2 magnetometers, J. Geophys. Res., 89, 786-800, 1984.

Ruohoniemi, J. M. and Baker, K. B.: Large-scale imaging of highlatitude convection with Super Dual Auroral Radar Network HF radar observations, J. Geophys. Res., 103, 20 797-20 811, 1998.

Ruohoniemi, J. M. and Greenwald, R. A.: The response of highlatitude convection to a sudden southward IMF turning, Geophys. Res. Lett., 25, 2913-2916, 1998.

Ruohoniemi, J. M., Shepherd, S. G., and Greenwald, R. A.: The response of the high-latitude ionosphere to IMF variations, J. Atmos. Sol.-Terr. Phys., 64, 159-171, 2002.
Saunders, M. A., Freeman, M. P., Southwood, D. J., Cowley, S. W. H., Lockwood, M., Samson, J. C., Farrugia, C. J., and Hughes, T. H.: Eastward propagation of a plasma convection enhancement following a southward turning of the interplanetary magnetic field, J. Geophys. Res., 97, 19373-19380, 1992.

Shepherd, S. G., Greenwald, R. A., and Ruohoniemi, J. M.: A possible explanation for rapid, large-scale ionospheric responses to southward turnings of the IMF, Geophys. Res. Lett., 26, 31973200, 1999.

Siscoe, G. L. and Huang, T. S.: Polar cap inflation and deflation, J. Geophys. Res., 90, 543-547, 1985.

Slinker, S. P., Fedder, J. A., Ruohoniemi, J. M., and Lyon, J. G.: Global MHD simulation of the magnetosphere for Nov. 24, 1966, J. Geophys. Res., 106, 361-380, 2001.

Todd, H., Cowley, S. W. H., Lockwood, M., Willis, D. M., and Lühr, H.: Response time of the high-latitude dayside ionosphere to sudden changes in the north-south component of the IMF, Planet. Space Sci., 36, 1415-1428, 1988. 